\title{
DISEÑO Y VALIDACIÓN DE LA ESCALA DE INTELIGENCIA ESPIRITUAL EN UNA MUESTRA DE TRABAJADORES DE SALUD. ICA, PERÚ
}

\author{
DESIGN AND VALIDATION OF A SCALE ON SPIRITUAL INTELLIGENCE IN A \\ SAMPLE OF HEALTH WORKERS. ICA, PERÚ
}

\author{
Bladimir Becerra-Canales ${ }^{1,2, a}$, Domizbeth Becerra-Huaman ${ }^{3,4, b}$. \\ 1. Dirección Regional de Salud. Ica, Perú. \\ 2. Universidad Autónoma de Ica, Perú. \\ 3. Universidad San Luis Gonzaga de Ica, Perú. \\ 4. Sociedad Científica de Estudiantes de Medicina de Ica (SOCEMI). Perú. \\ a. Doctor en Salud Pública. ORCID: oooo-00o2-2234-2189 \\ b. Estudiante de Medicina Humana. ORCID: oooo-0oo3- 2618-981X
}

DOI: https://doi.org/10.35563/rmp.v9i1.292

\section{Correspondencia:}

Bladimir Becerra Canales

Dirección: CC.HH La Angostura III

Etapa $\mathrm{H}-10$, Subtanjalla - Ica

Correo:

icapredica@gmail.com

Celular: 956690060

Contribuciones De Autoría: BBC: concepción y diseño del estudio, análisis estadístico, discusión y revisión final del manuscrito y DBH: recolección e interpretación de los datos y discusión.

Conflicto De Intereses: Los autores declaran no tener conflictos de interés. Financiamiento: Autofinanciado.

\section{Como Citar}

Becerra-Canales B. Becerra-Huamán $\mathrm{D}$. Diseño y validación de la escala de inteligencia espiritual en una muestra de trabajadores de salud. Ica, Perú. Rev méd panacea. 2020; 9(1): 23-30. doi: https://doi.org/10.35563/rmp.v9i1.292

Recibido: 10 - 02 - 2020

Aceptado: 25 - 02 - 2020

Publicado: 06 - 03 - 2020

\section{RESUMEN}

Objetivo: Diseñar y validar una escala para medir el perfil de inteligencia espiritual (EIE), en una muestra de trabajadores de salud. Materiales y métodos: Estudio observacional y analítico, fue desarrollado y modificado a través de dos estudios consecutivos ( $n=265$ y $n=265$, respectivamente), incluye dos fases: Cualitativa (Creación del instrumento) y cuantitativa (Evaluación de sus propiedades métricas). Resultados: El instrumento consta de 13 items, distribuidos en dos dominios, presentó un coeficiente Alfa de Cronbach de 0,82; con valores de correlación ítem-total de 0,46 hasta 0,60; el análisis factorial confirmatorio mostró una estructura de dos dimensiones que explicaba el $40,2 \%$ de la varianza total. El análisis bidimensional mostró correlación significativa $(p=0,00)$ y directa, entre la Dimensión I (Pensamiento Existencial) y la Dimensión II (Consciencia Trascendental en la Practica), además con ciertos comportamientos virtuosos: humildad para cambiar actitudes, iniciativa para solucionar problemas, compromiso, trato digno, buscar oportunidades para ayudar y empatía. Conclusiones: La EIE, presentó una buena consistencia interna, con correlaciones moderadas y significativas entre sus ítems y constituye un instrumento que puede ser usado para medir la inteligencia espiritual en trabajadores de salud y en diversos escenarios.

Palabras clave: Estudio de validacion, inteligencia espiritual, inteligencia, espiritualidad, escala.

\section{ABSTRACT}

Objective: To design and validate a scale to measure the profile of spiritual intelligence (EIE), in a sample of health workers. Materials and methods: An observational and analytical study was developed and modified through two consecutive studies ( $n=265$ and $n=265$, respectively), including two phases: Qualitative (Creation of the instrument) and quantitative (Evaluation of its metric properties). Results: The instrument consists of 13 items, distributed in two domains, presented a Cronbach alpha coefficient of 0.82 ; with item-total correlation values of 0.46 to 0.60 ; the confirmatory factor analysis showed a two-dimensional structure that explained $40.2 \%$ of the total variance. The two-dimensional analysis showed significant $(p=0.00)$ and direct correlation between Dimension I (Existential Thought) and Dimension II (Transcendental Consciousness in Practice), as well as certain virtuous behaviors: humility to change attitudes, initiative to solve problems, commitment, dignified treatment, seeking opportunities to help and empathy. Conclusions: The IEE presented a good internal consistency, with moderate and significant correlations between its items and constitutes an instrument that can be used to measure spiritual intelligence in health workers and in diverse scenarios.

Keywords: Validation study, spiritual intelligence, Intelligence, Spirituality, Scale. 


\section{INTRODUCCIÓN}

La inteligencia espiritual (IE), propuesta por Gardner $(1,2)$, en su teoría de inteligencias múltiples; en los últimos años ha recobrado mayor importancia y viene siendo estudiada por diversos investigadores (3-11).

Para, King (12); la IE, es el conjunto de capacidades mentales que contribuyen a la sensibilización, la integración y aplicación de los aspectos no materiales y trascendente de la existencia, lo que lleva a una profunda reflexión existencial, de mejora de significado, el reconocimiento de un ser trascendente, y el dominio de los estados espirituales. Esta definición concuerda con el enfoque de Gardner (2), quien considera que debiera llamarse más bien filosófica o existencial, ya que facultaría al ser humano para situarse a sí mismo con respecto al cosmos y buscar respuestas a los grandes interrogantes existenciales. La IE, nos ayuda a encontrar el sentido de la existencia humana y el significado de la vida $(13,14)$; de ahí, que la mayoría de autores, proponen el uso de este conocimiento para resolver problemas cotidianos, buscando una vida mejor (15).

Por su parte, Kwilecki (16), aplicó el modelo de Emmons (17), a un estudio de caso, confirmando empíricamente la observación de conductas ajustables a las dimensiones propuestas por este investigador, como la capacidad para la resolución de problemas que mostró el individuo a partir de conductas asociadas a este modelo de IE.

En ese contexto y con una visión más consciencial $(18,19)$, la IE es definida por el autor, como la conjunción de capacidades mentales que se ocupan de cuestiones existenciales, trascendentes $\mathrm{y} / \mathrm{o}$ consciénciales de la condición de Ser-Humano y su conexión con el universo; para desarrollar facultades que conducen a la sensibilización, humanización y autorrealización del Ser Humano auténticamente genuino; de ahí, que esta modalidad de inteligencia debiera llamarse también Inteligencia Consciencial (IC); porque, le permite al hombre reflexionar sobre sí mismo, ver el mundo material desde otras perspectivas, facilita el autoconocimiento y permite crear consciencia. Así mismo, considera que la IE o IC, es un constructo conformado por las siguientes dos dimensiones: i) Pensamiento Existencial (PE), se refiere a la capacidad de reflexionar crítica y profundamente acerca de existencia del ser humano, su posición dentro del cosmos, el significado de la vida y de la muerte entre otros temas existenciales para llegar a conclusiones propias $u$ originales (7). ii) Consciencia Trascendental en la Práctica (CTP), se refiere a la capacidad de saber escoger a la consciencia (Alma, Espíritu, Mónada cuántica, entre otros) sobre el ego, como el verdadero conductor de la vida, extrayendo su sabiduría para encontrar soluciones espirituales a los problemas de la práctica cotidiana.

En los últimos años, se han diseñados varios instrumentos dirigidos a medir la espiritualidad (20-22); sin embargo la mayoría de estos fueron desarrollados en idioma inglés y en poblaciones diferentes. Por su parte, King y DeCicco (7), desarrollaron un modelo, el resultado de este estudio se tradujo en un constructo para evaluar y medir el nivel de inteligencia espiritual, el autoinforme de inteligencia espiritual Inventario-24, SISRI-24.

Psicólogos, como Wolman (9) y Amram (3); argumentan que siendo la $\mathrm{IE}$, un tipo de inteligencia que promueve el desarrollo de Potencial Humano, es necesario volverlo operativo; tal como lo pretende el presente trabajo de investigación.

Desarrollar y cultivar Inteligencia IE, en todos los ámbitos es más necesaria que nunca, particularmente en el sector salud, permite formar servidores públicos de calidad humana, con altos niveles de humanización (Compasión, trato digno, empatía, solidaridad, respeto, responsabilidad, honestidad, entre otros); a nivel institucional permitiría humanizar los servicios de salud con "rostro humano", tener establecimientos sanitarios de calidad y un sistema de salud más efectivo; de ahí, que abordar la temática propuesta y generar un instrumento de medición de la $\mathrm{IE} / \mathrm{IC}$, resulta relevante en estos tiempos de crisis de consciencia; dada la incapacidad de muchos seres humanos de reconocer su verdadera esencia, reflejada en manifestaciones de carencia moral y falta de ética como pautas y paradigmas de la sociedad que tenemos hoy en día; realidad de la cual, no podemos ser indiferentes.

Considerando este planteamiento el objetivo del estudio, fue diseñar y validar una escala para medir el perfil de inteligencia espiritual, en una muestra de trabajadores de salud de Ica, Perú

\section{MATERIALES Y MÉTODOS}

\section{Diseño y participantes.}

Reuniendo los elementos considerados hasta aquí, se planteó un estudio observacional, transversal, prospectivo y analítico; de diseño, validación de instrumentos documentales, durante el primer semestre del año 2019, involucrando a trabajadores de la Dirección Regional de Salud Ica, Red de Salud Ica, Hospitales y Centros de Salud. Se estimó un tamaño muestral mínimo para validar una escala de 15 ítems en 265 participantes en la primera y segunda fase $(n=265$ y $n=265)$, considerando, por lo menos tener 16 unidades muestrales por ítem (23), que fueron seleccionadas por muestreo accidental. Se incluyeron a trabajadores de áreas administrativas y asistenciales, que se encuentre en el momento de la aplicación del instrumento y que deseen participar en el estudio; se excluyeron trabajadores ausentes y que no deseaban participar en el estudio

\section{Desarrollo y validación de la EIE.}

Para evaluar la IE/IC, se construyó una escala que Ilamamos Escala de Inteligencia Espiritual (EIE), según un modelo teórico propio (explicado en la introducción) basado en las publicaciones previas (3-11). El procedimiento de diseño y validación se realizó en dos fases $(24,25)$ : 
Fase cualitativa: Que corresponde a la creación del instrumento (validez de contenido), incluye: a) Revisión del concepto de la literatura: El cual consistió en la revisión del conocimiento disponible que se tiene hasta el momento respecto al concepto de la variable en estudio, concluyendo que la misma se encontraba parcialmente definida. b) Formulación de los ítems del instrumento: Teniendo en cuenta la circunstancia que el concepto de la variable se encontraba parcialmente definida, algunos ítems de los 46 iniciales se formularon a partir de la revisión de la literatura y/o modificando reactivos de otros instrumentos. c) Evaluación del contenido por jueces: Se eligió a un grupo multidisciplinario de jueces (05 profesionales) pertenecientes a distintos campos del conocimiento a fin de evitar percepciones sesgadas y opiniones subjetivas acerca del tema en proceso de evaluación. La tarea de los jueces fue revisar los ítems ya construidos en términos de suficiencia, pertinencia y claridad con la que están redactados, lo que permitió reducir el número de ítems del instrumento a 30 reactivos. d) Prueba piloto: Los ítems del instrumento, fueron formulados en dos sentidos, la mitad de ellos en sentido favorable y la otra desfavorable respectivamente. El instrumento así construido fue aplicado a 265 trabajadores de salud; en esta fase se volvió a evaluar la claridad con la que están redactados los ítems, mejorando la sintaxis y semántica de alguno de ellos.

Fase cuantitativa: Que corresponde a la evaluación de las propiedades métricas, incluye: a) Análisis de la consistencia: Se procedió a explorar la correlación de cada uno de los ítems respecto del total, para evaluar la idoneidad de estos ítems según lo cual se quedan o no en el instrumento. Se obtuvo un valor global preliminar de la consistencia interna mediante el cálculo del Alfa de Cronbach, el cual resultó 0,8. b) Análisis de la variabilidad y matriz de correlación: En esta fase de la reducción de los ítems, fueron eliminados 10 ítems más, de tal modo que nos quedamos únicamente con 20, para tal efecto se ordenaron todos los ítems según el índice de correlación que guardan con el resultado total de mayor a menor, de tal modo que los últimos 10 ítems que tuvieron la menor magnitud de correlación con el puntaje total y aquellos que expresaron menor grado de variabilidad fueron eliminados. c) Análisis factorial confirmatorio: Si bien, ya teníamos definidos cualitativamente a los ítems que iban a conformar cada una de las dimensiones, se realizó el análisis factorial confirmatorio, en este procedimiento se perdió 7 ítems más, quedando el instrumento estructurado con 13 ítems, distribuidos en dos dimensiones.

Análisis estadísticos. Se describieron las características generales de la población objeto de estudio mediante frecuencias absolutas y relativas para las variables categóricas, así como medidas de tendencia central y dispersión para las variables numéricas.

Se realizó un análisis de la variabilidad de los ítems, considerándose como elevados los valores superiores a
0,93; dado que, al tratarse de una escala de tipo Likert, se acepta un valor mínimo de 0,20 para poder afirmar que los ítems discriminan (26); así mismo un análisis de la matriz de correlación de los ítems e ítem total, mediante el coeficiente de correlación de Pearson (considerándose como apropiados los valores superiores a 0,50). Previo al análisis factorial, se determinó la existencia de relaciones significativas entre las variables, con el Test de Esfericidad de Bartlett y el test de Kaiser-Meyer-Olkin (KMO), tomando como un correcto ajuste de la muestra promedio y por ítem cuando los valores fueron superiores a 0,7 (27).

Se incluyó un análisis de fiabilidad para escalas y la consistencia interna fue medida con el Alpha de Cronbach; garantizando que estos índices superen el mínimo recomendado por la literatura $(, 70)$ para ser considerado un instrumento confiable (28); Se hicieron correlaciones con las consecuencias naturales del concepto medido, considerando un intervalo de confianza al $95 \%$ y a todo valor de $p<0,05$ como estadísticamente significativo. Para el procesamiento de los datos se usó el paquete estadístico "Statistical Package for the Social Sciences" para Windows versión 24.0 en Español.

Aspectos éticos. Se tuvo en cuenta la Declaración de Helsinki (World Medical Association, 2013), que establece los principios éticos fundamentales para la investigación con seres humanos. Se solicitó el permiso institucional y se indicó al trabajador de salud que su participación era voluntaria y anónima, obteniéndose el consentimiento verbal.

\section{RESULTADOS.}

Para validar las propiedades métricas, el instrumento fue aplicado en una segunda ocasión a 265 trabajadores de salud.

La Tabla 1, presenta la descripción de las características generales de los trabajadores de salud, la mayoría eran de condición laboral nombrado (56.2\%); asistenciales (52.1\%) y procedían de centros de salud (39.6\%); el promedio de edad fue de 42 años.

Tabla1. Descripción de las características del trabajador de salud

\begin{tabular}{llcc}
\hline \multicolumn{1}{c}{ Variables de caracterización } & № & $\%$ \\
\multirow{3}{*}{ Sexo } & Masculino & 120 & 45.3 \\
Condición laboral & Femenino & 145 & 54.7 \\
& Nombrado & 149 & 56.2 \\
Grupo ocupacional & Contratado & 116 & 43.8 \\
& Administrativo & 127 & 47.9 \\
& Asistencial & 138 & 52,1 \\
\multirow{2}{*}{ Centro laboral } & Dirección Regional de Salud Ica & 73 & 27.5 \\
& Red de Salud Ica & 49 & 18.5 \\
\multirow{2}{*}{ Edad en años } & Hospitales & 38 & 14.4 \\
& Centros de Salud & 105 & 39.6 \\
\hline
\end{tabular}

Nota: $M$ = Media; $\mathrm{DE}=$ Desviación estándar. 
La Tabla 2, presenta el análisis de variabilidad de los ítems, el mismo que reveló que la capacidad discriminante de los ítems, es en general muy elevada encontrando índices de variabilidad superiores a 0.93 ; con una mediana de 1.13 se constata que todos los ítems son muy homogéneos en términos de varianza, siendo adecuados para discriminar entre sujetos con valores distintos, en la variable medida. El análisis de la matriz de correlación (Coeficiente de Pearson), reveló correlaciones positivas entre los ítems, con valores de correlación ítems-total, desde .50 a .60 $(p<0.00)$.

Tabla2. Análisis de la variabilidad de los ítems y matriz de correlación

\begin{tabular}{|c|c|c|c|}
\hline № & Ítems & Varianza & $\begin{array}{l}\text { Correlación } \\
\text { (Suma) }\end{array}$ \\
\hline 1 & $\begin{array}{l}\text { He reflexionado profundamente si existe o no una } \\
\text { Consciencia (Espíritu/Alma) }\end{array}$ & 1.26 & .56 \\
\hline 2 & $\begin{array}{l}\text { A menudo pienso en la recompensa espiritual que podría } \\
\text { recibir si hago bien mi trabajo. }\end{array}$ & 1.15 & .51 \\
\hline 3 & $\begin{array}{l}\text { Escojo a la Mente sobre la Consciencia para que la vida se } \\
\text { conduzca mejor. }\end{array}$ & .93 & .54 \\
\hline 4 & $\begin{array}{l}\text { Pienso que todos somos uno con el universo y que } \\
\text { estamos conectados unos a otros, en forma de una red }\end{array}$ & 1.34 & .58 \\
\hline 5 & $\begin{array}{l}\text { El despertar de la Consciencia, es una práctica constante } \\
\text { en mi vida. }\end{array}$ & .93 & .59 \\
\hline 6 & $\begin{array}{l}\text { He reflexionado profundamente, acerca de lo que puede } \\
\text { haber más allá de la muerte del cuerpo físico. }\end{array}$ & .99 & .54 \\
\hline 7 & $\begin{array}{l}\text { Busco constantemente encontrar respuesta a los } \\
\text { misterios de la vida, el mundo espiritual entre otras }\end{array}$ & 1.29 & .60 \\
\hline 8 & $\begin{array}{l}\text { Me considero un ser espiritual viviendo una experiencia } \\
\text { humana. }\end{array}$ & 1.24 & .60 \\
\hline 9 & $\begin{array}{l}\text { Asumo que la Consciencia, es la base de todo cuanto } \\
\text { existe. }\end{array}$ & 1.06 & .60 \\
\hline 10 & $\begin{array}{l}\text { Me defino a mí mismo por mi cuerpo físico y/o } \\
\text { personalidad. }\end{array}$ & 1.15 & .50 \\
\hline 11 & $\begin{array}{l}\text { Con frecuencia me he preguntado sobre si la vida misma } \\
\text { tiene un sentido. }\end{array}$ & 1.00 & .57 \\
\hline 12 & $\begin{array}{l}\text { Valoro a la Consciencia, como más importante que el } \\
\text { cuerpo físico. }\end{array}$ & 1.05 & .52 \\
\hline 13 & $\begin{array}{l}\text { He sacado mis propias conclusiones/teorías en temas } \\
\text { como la vida y la existencia misma. }\end{array}$ & .95 & .50 \\
\hline & SUMA & 75.28 & \\
\hline
\end{tabular}

Se realizó el Análisis Factorial Exploratorio y el test de esfericidad de Bartlett arrojo un estadístico de 686.509; con un $\mathrm{p}=0,000$; la medida de adecuación muestral $\mathrm{KMO}$ fue de .871; lo cual aconseja su factorización ya que nos permiten afirmar que existen correlaciones significativas entre las variables; el Análisis Factorial Confirmatorio, reveló una estructura de dos factores, que explicaron el $40.2 \%$ de la varianza total del cual $31,93 \%$ lo explica el factor 1 y $8,20 \%$ el factor 2 (Tabla 3); de este modo se observa además en el gráfico de sedimentación, que el factor 1, aporta la mayor variabilidad, mostrando la proporción de varianza que explica cada uno de los componentes en el resultado total (Figura 1). En la tabla 4, se presentan las cargas factoriales obtenidas por los 13 reactivos de la versión final de la EIE, cuya distribución por dimensiones fue la siguiente: 7 reactivos en el primer factor y 6 en el segundo. Al analizar la agrupación de los reactivos por su carga factorial y contrastarlos con el modelo teórico propuesto por el autor, se determinó que los reactivos agrupados en el primer factor $(1,4,6,7,11$, 12 y 13) pertenecen a la dimensión Pensamiento Existencial (PE) y el segundo factor agrupó a los reactivos 2, 3, 5, 8, 9 y 10, que corresponden a la dimensión Consciencia Trascendental en la Practica (CTP).
Tabla3. Análisis Factorial Confirmatorio

\begin{tabular}{|c|c|c|c|c|c|c|}
\hline \multirow{4}{*}{ Componente } & \multicolumn{5}{|c|}{ Varianza total explicada } & \\
\hline & \multicolumn{3}{|c|}{ Autovalores iniciales } & \multicolumn{3}{|c|}{$\begin{array}{c}\text { Sumas de las saturaciones al cuadrado } \\
\text { de la extracción }\end{array}$} \\
\hline & & $\%$ de la & $\%$ & & $\%$ de la & $\%$ \\
\hline & Total & varianza & acumulado & Total & varianza & acumulado \\
\hline 1 & 4,15 & 31,926 & 31,926 & 4,15 & 31,926 & 31,926 \\
\hline 2 & 1,066 & 8,202 & 40,128 & 1,066 & 8,202 & 40,128 \\
\hline 3 & 1,002 & 7,706 & 47,834 & & & \\
\hline 4 & 0,93 & 7,15 & 54,984 & & & \\
\hline 5 & 0,864 & 6,642 & 61,626 & & & \\
\hline 6 & 0,821 & 6,315 & 67,942 & & & \\
\hline 7 & 0,747 & 5,747 & 73,688 & & & \\
\hline 8 & 0,687 & 5,287 & 78,975 & & & \\
\hline 9 & 0,649 & 4,991 & 83,967 & & & \\
\hline 10 & 0,603 & 4,64 & 88,607 & & & \\
\hline 11 & 0,521 & 4,005 & 92,612 & & & \\
\hline 12 & 0,485 & 3,734 & 96,346 & & & \\
\hline 13 & 0,475 & 3,654 & 100 & & & \\
\hline
\end{tabular}

Tabla4. Cargas factoriales de reactivos en los tres factores de la EIE

\begin{tabular}{|c|c|c|c|}
\hline № & Items & Factor 1 & Factor 2 \\
\hline 6 & $\begin{array}{l}\text { He reflexionado profundamente, acerca de lo que puede } \\
\text { haber más allá de la muerte del cuerpo físico. }\end{array}$ & 0,711 & \\
\hline 4 & $\begin{array}{l}\text { Pienso que todos somos uno con el universo y que } \\
\text { estamos conectados unos a otros, en forma de una red }\end{array}$ & 0,639 & \\
\hline 1 & $\begin{array}{l}\text { He reflexionado profundamente si existe o no una } \\
\text { Consciencia (Espíritu/Alma) }\end{array}$ & 0,602 & \\
\hline 13 & $\begin{array}{l}\text { He sacado mis propias conclusiones y/o teorías en temas } \\
\text { como la vida y la existencia misma. }\end{array}$ & 0,565 & \\
\hline 7 & $\begin{array}{l}\text { Busco constantemente encontrar respuesta a los } \\
\text { misterios de la vida, el mundo espiritual y a preguntas }\end{array}$ & 0,484 & \\
\hline 12 & $\begin{array}{l}\text { Valoro a la Consciencia, como más importante que el } \\
\text { cuerpo físico. }\end{array}$ & 0,484 & \\
\hline 11 & $\begin{array}{l}\text { Con frecuencia me he preguntado sobre si la vida misma } \\
\text { tiene un sentido. }\end{array}$ & 0,476 & \\
\hline 3 & $\begin{array}{l}\text { Escojo a la Mente sobre la Consciencia para que la vida se } \\
\text { conduzca mejor. }\end{array}$ & & 0,371 \\
\hline 2 & $\begin{array}{l}\text { A menudo pienso en la recompensa espiritual que podría } \\
\text { recibir si hago bien mi trabajo. }\end{array}$ & & 0,73 \\
\hline 8 & $\begin{array}{l}\text { Me considero un ser espiritual viviendo una experiencia } \\
\text { humana. }\end{array}$ & & 0,704 \\
\hline 10 & $\begin{array}{l}\text { Me defino a mí mismo por mi cuerpo físico y/o } \\
\text { personalidad. }\end{array}$ & & 0,575 \\
\hline 5 & $\begin{array}{l}\text { El despertar de la Consciencia, es una práctica constante } \\
\text { en mi vida. }\end{array}$ & & 0,571 \\
\hline 9 & $\begin{array}{l}\text { Asumo que la Consciencia, es la base de todo cuanto } \\
\text { existe. }\end{array}$ & & 0,499 \\
\hline
\end{tabular}

Figura1. Gráfico de sedimentación posterior al análisis de componentes principales con los 13 items incluidos.

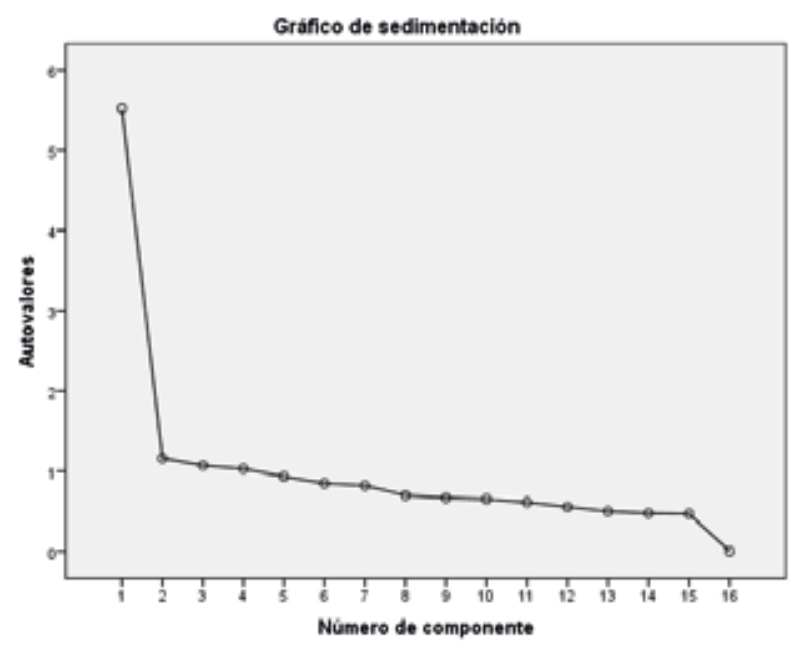


La Tabla 5, presenta el análisis de fiabilidad, la misma que se realizó con el Alfa de Cronbach, el cual se calculó mediante el método de la varianza de los ítems; en la tabla 4, se observa los 13 ítems incluidos; y el puntaje específico para cada ítem si este fuera retirado, que en todos los casos es mayor a .82. Asimismo, la correlación elemento-total corregida fue superior a .40 . La consistencia interna global fue alta (Alfa $=.82$ ), así como para la dimensión PE (.73) y la dimensión CTP (.72); lo cual indica que se trata de un instrumento fiable que ara mediciones estables y consistentes.

Tabla5. Análisis de consistencia interna mediante coeficiente alfa de Cronbach de la EIE

\begin{tabular}{ccc} 
№ & $\begin{array}{c}\text { Correlación elemento } \\
\text { total corregida }\end{array}$ & $\begin{array}{c}\text { Alfa si el ítems se } \\
\text { elimina }\end{array}$ \\
\hline Item01 & .47 & .82 \\
Item02 & .41 & .83 \\
Item03 & .46 & .82 \\
Item04 & .48 & .82 \\
Item05 & .51 & .82 \\
Item06 & .45 & .82 \\
Item07 & .50 & .82 \\
Item08 & .51 & .82 \\
Item09 & .51 & .82 \\
Item10 & .40 & .83 \\
Item11 & .48 & .82 \\
Item12 & .43 & .82 \\
Item13 & .41 & .83 \\
\hline
\end{tabular}

Nota: Alfa = Alfa de Cronbach.

La Tabla 6, presenta la validez de criterio, para realizar el análisis de la misma, no se disponía de un instrumento para medir la IE con una visión consciencial; por tal razón, se realizaron correlaciones con las consecuencias naturales del concepto medido, considerando para el análisis ciertos comportamientos virtuosos, que se derivan de la variable en estudio; como humildad para cambiar actitudes, iniciativa para solucionar problemas, compromiso, trato digno, buscar oportunidades para ayudar y empatía.; demostrando que las correlaciones fueron significativas en la mayoría de los casos, además de ser directas.

Tabla6. Validez concurrente

\begin{tabular}{lccccc}
\multicolumn{1}{c}{ Comportamientos virtuosos } & \multicolumn{2}{c}{ PE } & \multicolumn{2}{c}{ CTP } \\
& $\begin{array}{l}\text { Correlación } \\
\text { de Pearson }\end{array}$ & p-valor & $\begin{array}{c}\text { Correlación } \\
\text { de Pearson }\end{array}$ & p-valor \\
Humildad para cambiar actitudes. & .12 & .044 & .13 & .042 \\
Iniciativa para solucionar problemas & .12 & .043 & .22 & .000 \\
Necesidad de compromiso institucional & .13 & .040 & .20 & .001 \\
Reconocer que no siempre trata bien a los usuarios & .21 & .000 & .22 & .000 \\
Buscar oportunidades para ayudar. & .19 & .002 & .16 & .012 \\
Ponerse en el lugar del paciente & .16 & .012 & .26 & .000 \\
PE & & & .68 & .000 \\
CTP & .68 & .000 & & \\
\hline
\end{tabular}

Nota: $\mathrm{PE}=$ Pensamiento Existencia; $\mathrm{CTP}=$ Consciencia Trascendental en la Practica

\section{DISCUSIÓN}

La revisión de la literatura, confirma que actualmente existen escasos instrumentos de medición del constructo
IE; la mayoría de ellos fueron desarrollados, validados y/o adaptados en contextos canadienses y/o anglosajones $(7,29)$ y muy pocos latinoamericanos $(30)$. Lo que conlleva a una serie de problemas de diversidad cultural en un constructo tan complejo (31).

Por tal razón, urge la necesidad de proponer a la comunidad científica latinoamericana instrumentos fiables y contextualizados que evalúen la $\mathrm{IE}$, en una visión más consciencial $(18,19)$ y operativa $(3,9)$.

En ese orden de ideas, el estudio tuvo como objetivo generar una escala de medida respecto a la IE en una muestra de trabajadores del área de salud, que posea validez de contenido y propiedades métricas aceptables. Esta versión del instrumento facilitará futuras investigaciones sobre el constructo IE/IC y sus posibles asociaciones con otras variables de interés científico, para empezar a crear consciencia de cara a la humanización de la sociedad y de las instituciones públicas.

Como muestran los resultados de la evaluación de las propiedades métricas; la EIE, presenta una consistencia interna aceptable (.82), con correlaciones moderadas y significativas entre sus ítems. Las pruebas psicométricas utilizadas para justificar el análisis factorial fueron el test de esfericidad de Bartlett (que evalúa si la matriz de correlaciones es una matriz de identidad) y la medida de adecuación muestral KMO (que compara los coeficientes de correlación observados con los coeficientes de correlación parcial). En nuestro estudio, el test de esfericidad de Barlett tuvo un valor significativo y la medida de adecuación muestral KMO (.876). En base a estos dos resultados favorables, se procedió al análisis factorial de la EIE, el cual confirmó el supuesto teórico según el cual se basó el diseño de la escala (dos dimensiones), donde la dimensión 1 explica la mayor parte de la varianza recogida respecto a la dimensión 2 . Resumiendo, nos encontramos con una estructura factorial consistente con ítems que pueden medir adecuadamente la variable estudiada; que además cuenta con suficiente evidencia científica, que apoye la IE, como constructo claramente definido (3-11).

El instrumento estructurado, consta de 13 items, distribuidos en dos dimensiones: Pensamiento Existencial-PE (Ítems 1, 4, 6, 7, 11, 12 y 13) y Consciencia Trascendental en la Practica-CTP (Ítems 2, 3, 5, 8, 9 y 10), con cinco opciones de respuesta (Nada cierto, un poco cierto, algo cierto, bastante cierto, muy cierto), con puntaje del 1 al 5, considerando que el ítems 3 y 10 se han redactado en sentido negativo

La EIE, tiene una puntuación global en forma numérica, con calificaciones que van desde los 13 hasta los 65 puntos, donde el puntaje <39 equivale a una IE No saludable o inadecuada, de 39-52 IE por mejorar y $>52$ puntos IE saludable o adecuada; la escala presenta dos dimensiones: i) PE (puntaje del 7 al 35); ii) CTP (puntaje del 6 al 30); las puntuaciones solo denotan intensidad, no es 
el objetivo de la escala global ni de las dimensiones tener una función discriminante. Como los ítems 3 y 10 tienen orientación negativa, deben ser invertidos a la hora de sumar la puntuación. Es importante precisar que el instrumento de medición documental mide una variable subjetiva, por lo tanto puede variar de acuerdo al contexto donde se aplique.

En cuanto a las limitaciones del estudio, consideramos que el instrumento de medición documental generado mide una variable subjetiva, la cual se trata de un pensamiento abstracto, sin embargo es importante como medida de IE; toda vez que, desarrollar y cultivar IE/IC, es más necesaria que nunca, en estos tiempos de crisis de consciencia, dada la incapacidad del ser humano de reconocer su verdadera esencia, reflejada en la deshumanización de la sociedad. Estudiar esta variable particularmente en el ámbito sanitario, nos permitiría conocer una realidad pocas veces explorada y en función a los resultados planificar y ejecutar acciones y/o planes de mejora, con la finalidad de contribuir a humanizar los servicios de salud. Por otro lado la falta de sinceridad por parte de algunos sujetos a la hora de emitir respuestas, constituye una limitación importante, sin embargo esta puede ser superada con un adecuado control de calidad de las fichas y una correcta aplicación de la encuesta heteroadministrada; finalmente la utilización de una muestra recopilada de forma accidental y no aleatoria, puede ser considerada como una debilidad.

Recomendamos administrar la EIE, a otra muestra de participantes para realizar el proceso de validación cruzada. De igual forma, se debe llevar a cabo un análisis de factores confirmatorio con el propósito de examinar la estructura factorial identificada en este estudio y finalmente recomendamos que la EIE, se valide en poblaciones de otros países, para auscultar sus propiedades psicométricas.

Los resultados obtenidos de la EIE, demuestran índices de confiabilidad y validez dentro de lo esperado; esto fue evidenciado al observar la consistencia interna del instrumento final. De igual forma, la validez de constructo del instrumento fue examinada a través de un análisis de variabilidad, correlación y factores confirmatorio, las propiedades psicométricas de la escala final demuestran que el instrumento es apropiado, sin embargo puede ser mejorado, por lo cual se recomienda su uso en futuras investigaciones. 


\section{REFERENCIAS BIBLIOGRÁFICAS}

1. Gardner H. Frames of Mind. The Theory of Multiple Intelligences. Nueva York, Basic Books. (Versión castellana (2001): Estructuras de la Mente. La Teoría de las Inteligencias Múltiples. México, FCE; 1983.

2. Gardner H. Intelligence Reframed: Multiple Intelligences for the 21st Century. Nueva York, Basic Books. (Versión castellana (2001): La Inteligencia Reformulada. Las Inteligencias Múltiples en el Siglo XXI. Barcelona, Paidos; 1999.

3. Amram Y. The seven dimensions of spiritual intelligence: An ecumenical, grounded theory. Paper presented at the 115th Annual Conference of the American Psychological Association, San Francisco, California, USA. [Internet]. Retrieved from; 2007. [citado el 9 de Agosto de 2019]. Disponible en: http://www.yosiamram.net/docs/7_Dimensions_of_SI_ APA_confr_paper_Yosi_Amram.pdf

4. Anbugeetha, D. An analysis of the spiritual intelligence self-report inventory (SISRI). International Journal of Management (IJM). 2015; 6(7), 25-36. ID: 10120150607004

5. Emmons R. Is spirituality an intelligence. The International Journal for the Psychology of Religion. 2000; 10(1): 3-26. DOI:10.1207/S15327582IJPR1001_2

6. Golovina $O$. The spiritual intelligence as an integrating factor of human consciousness. Zenodo. 2016; 52, 44-52. DOI:10.5281/zenodo.44709

7. King DB, DeCicco TL. A viable model and self-report measure of spiritual intelligence. The International Journal of Transpersonal Studies. 2009; 28, 68-85. DOI:10.24972/ijts.2009.28.1.68

8. Mahasneh AM, Shammout NA, Alkhazaleh ZM, Al-Alwan AF, Abu-Eita JD. The relationship between spiritual intelligence and personality traits among Jordanian university students. Psychol Res Behav Manag. 2015; 8: 89-97. DOI: 10.2147/PRBM.S76352

9. Wolman R. Thinking with your soul. Spiritual intelligence and why it matters. New York, NY: Harmony Books; 2001.

10. Sokhandani M, Jalili S, Mohammadalipoor Z. Role of spiritual intelligence in defensive styles of nursing students. International Journal of Interdisciplinary Studies and Research. 2019; 6(4), 73-82. Recuperado de:

https://www.sid.ir/en/journal/ViewPaper.aspx?id=4846 39

11. Zohar D, Marshall I. Spiritual intelligence, the ultimate intelligence, SQ. London, UK: Bloomsbury; 2000.

12. King D. B. Rethinking claims of spiritual intelligence: $A$ definition, model, \& measure. Unpublished master's thesis, Trent University, Peterborough, ON, Canada 2008. [citado el 12 de julio de 2019]. Disponible en: https://docplayer.net/4796647-Rethinking-claims-of-s piritual-intelligence-a-definition-model-and-measure. html

13. Emmons R. The psychology of ultimate concerns. New York: Guilford Press.1999.

14. Halama P, Striženec M. Spiritual, existential or both?
Theoretical considerations on the nature of "higher" intelligences. Studia Psychologica. 2004; 46(3): 239-253. Recuperado de:

https://www.researchgate.net/publication/28844634 6_Spiritual_existential_or_both_Theoretical_considera tions_on_the_nature_of_higher_intelligences

15. Antunes R, Silva A, Oliveira J. Spiritual Intelligence Self-Assessment Inventory: Psychometric properties of the Portuguese version of SISRI-24, Journal of Religion, Spirituality \& Aging. 2017; 30: 12-14. DOI: 10.1080/15528030.2017.1324350

16. Kwilecki S. Spiritual intelligence as a theory of individual religion: A case application, The International Journal for the Psychology of Religion. 2000; 10(1): 35-46. https://doi.org/10.1207/S15327582IJPR1001_4

17. Emmons R. Spirituality and intelligence: Problems and prospects. Journal The International Journal for the Psychology of Religion. 2009; 10,57-64. https://doi.org/10.1207/S15327582IJPR1001_6.

18. Bonilla E. Conexión mente-cuerpo, fenómenos parapsicológicos y curación espiritual. Invest Clin. 2010; 51(2): 209-238. Recuperado de: http://ve.scielo.org/scielo.php?script=sci_arttext\&pid =S0535-51332010000200005

19. Carrillo E. Amor: Vida y Consciencia, 2012. Recuperado de: https://despertadhumanidad.wordpress.com/2012/1 2/10/amor-vida-y-consciencia-emilio-carrillo-libro-gr atis/

20. Baldacchino D, Buhagiar A. Psychometric evaluation of the Spiritual Coping Strategies scale in English, Maltese, backtranslation and bilingual versions. Journal of Advanced Nursing. 2003; 42(6):558-570. DOI: 10.1046/j.1365-2648.2003.02659.x

21. Hall $D$, Meador $K$, Koenig $H$. Measuring religiousness in health research: review and critique. Journal of Religion and Health. 2008; 47(2), 134-163. DOI: 10.1007/s10943-008-9165-2.

22. Fetzer Institute/National Institute on Aging Working Group. Multidimensional Measurement of Religiousness/Spirituality for Use in Health Research. Kalamazoo, Michigan: Fetzer Institute. Recuperado de: $\quad$ https://www.gem-measures.org DownloadMeasure. 1999.

23. Mayta-Tristán $P$, Mezones-Holguín E, Pereyra-Elías R, Montenegro-Idrogo JJ, Mejia C, Dulanto-Pizzorni A. Diseño y validación de una escala para medir la percepción sobre el trabajo en el primer nivel de atención en estudiantes de medicina de Latinoamérica. Rev Peru Med Exp Salud Pública. 2013; 30(2):190-6.

DOI: http://dx.doi.org/10.17843/rpmesp.2013.302.190

24. Supo J. Validación de instrumentos de medición documentales, curso en vídeo para descarga inmediata; 2010. Recuperado de: https://validaciondeinstrumentos.com/carta

25. Supo J. Cómo validar un instrumento - La guía para 
validar un instrumento en 10 pasos; 2013. Recuperado de:

http://www.cua.uam.mx/pdfs/coplavi/s_p/doc_ng/valid acion-de-instrumentos-de-medicion.pdf

26. Barbero M. Psicometría II. Métodos de elaboración de escalas. Madrid: Uned; 1993.

27. Pett M, Lackey N, Sullivan J. Making sense of factor analysis: the use of factor analysis for instrument development in health care research. California: Sage Publication; 2003. DOI:

https://dx.doi.org/10.4135/9781412984898

28. Campo-Arias A, Oviedo $H$. Propiedades psicométricas de una escala: La consistencia interna. Revista de Salud Pública. 2008; 10(5), 831-839. Recuperado de: https://www.redalyc.org/pdf/422/42210515.pdf
29. Skrzypińska K. Polish adaptation of Spiritual Intelligence Self-Report Inventory (SISRI-24). Polonia: ResearchGate. 2016; Recuperado de: https://www.researchgate.net/publication/30809757 4_Polish_adaptation_of_Spiritual_Intelligence_Self-Re port_Inventory_SISRI-24

30. Antunes R, Silva A, Oliveira J. Spiritual Intelligence Self-Assessment Inventory: Psychometric properties of the Portuguese version of SISRI-24, Journal of Religion, Spirituality \& Aging. 2017; 30,12-14 DOI: 10.1080/15528030.2017.1324350

31. Arias $R$, Lemos V. Una aproximación teórica y empírica al constructo de inteligencia espiritual. Enfoques. 2017; 27(1), 79-102. Recuperado de: http://www.scielo.org.ar/scielo.php?script=sci_arttext \&pid=S1669-27212015000100005\&lng=es\&tIng=es

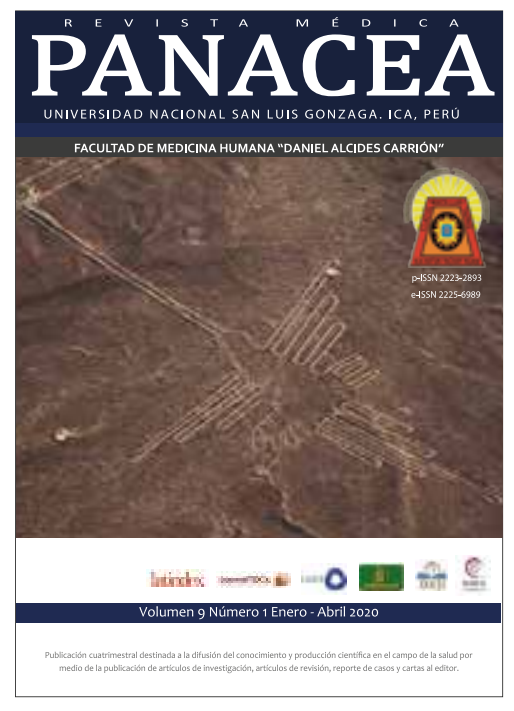

